\title{
Influence of Varying Thermodynamic Magnitudes on the Acoustic Levitation of Particles in a Single Axis Ultrasonic Levitator
}

\author{
Sebastian Baer, Cemal Esen, and Andreas Ostendorf
}

\begin{abstract}
This work describes the development and analysis of a single-axis acoustic levitator, which consists of a $38,5 \mathrm{kHz}$ Langevin-type piezoelectric transducer with a concave radiating surface and a concave reflector under increased temperatures and pressures. The levitator design is implemented in a Single-Droplet Optical Cell for levitation processes under varying atmospheres. The advantages of acoustic levitation of small droplets under increased temperatures and pressure combined with spectroscopic applications enable novel experiments possibly relevant to the fields of chemical engineering, planetary science, metrology and combustion chemistry. In this investigation the optimal transducer-reflector distances of a single axis ultrasonic levitator for various temperatures and pressures of different gases are simulated with a FEM-method using a modified Gorkov equitation and experimentally verified.
\end{abstract}

Index Terms-Acoustic levitation, high pressure application, single axis ultrasonic levitator, thermodynamic application.

\section{INTRODUCTION}

In the last years, acoustic levitation has been used in a broad range of applications including analytical chemistry, material science and medical chemistry [1].

Especially the knowledge of equilibrium states of binary mixtures is of great interest in the fields of chemical engineering. The cost efficient investigation of material compositions in industrial process engineering using small amounts of materials is eligible. Commonly used sampling techniques like gas chromatographic analysis are used for concentration measurements. These techniques face problems if the extraction of the sample changes its condition. Furthermore, the use of small sample volumes leads to many problems concerning the handling, a higher chance of contamination, sorption and other processes caused by the use of container walls that interact with the observed sample. Different levitation methods have been studied by [2], such as optical [3], electrostatic [4], aerodynamic [5], ion [6], diamagnetic [7] and acoustic levitation. Among these techniques, acoustic levitation has the advantage of not requiring any specific physical properties of the sample, such as a specific electrical charge, a certain refractive index or

Manuscript received November 23, 2013; revised January 6, 2014. The project was funded by the German Research Association (DFG) under No. Schw184/43-1. and by the Research School Plus of the Ruhr Universität Bochum.

The authors are with Applied Laser Technology of the Ruhr Universität Bochum, Universitätsstr. 150, 44801 Bochum, Germany (e-mail: baer@lat.rub.de, esen@lat.rub.de, andreas.ostendorf@rub.de). transparency.

In 1933 the concept of levitation by acoustic radiation pressure was first proposed by [8], [9] derived the theoretical background in 1934. A typical acoustic levitator generates an acoustic standing wave between a transducer and a reflector separated by a multiple integer number of half wavelengths of the acoustic wave. The small samples are levitated against gravity by the pressure forces and tend towards a stable equilibrium position close to the acoustic nodal points. Acoustic levitation in combination with analytical applications like Raman spectroscopy is a very powerful technique in analytical chemistry or process engineering and has been used in a lot of applications. This combination was first introduced by [10], [11] to observe the solidification process of acoustically levitated solutions. Acoustic levitation has been used by [12] for simplifying the handling of micro samples in laser spectroscopy. By monitoring the malaria pigment hemozoin in vital blood cells and online monitoring of environmentally stressed living cells [13], [14] applied this technique into the field of biospectroscopy. Spectroscopy in combination with acoustic levitation has been used for airborne chemistry and protein crystallization by [2], [15]. The backscattered Raman signal was used by [16] for the chemical analysis of levitated drops. [17] showed theoretically and experimentally the possibility of Raman scattering on deformed acoustically levitated droplets by a scattering angle of $90^{\circ}$. A method of calculation of the acoustic radiation pressure based on the boundary element method was presented by [18] and the influence of the acoustic field on the external flow is described in [19].

One of the problems during working with acoustic levitation is the instability of the levitated particle. In commonly used acoustic levitators, acoustic radiation forces that act on the particle are strong, generating high axial and radial stability shown by [20]. However, the acoustic radiation forces get weak if the conditions for a standing ultrasonic wave fail by changing the sound velocity, resulting in oscillations up to no levitation of the sample. The effects of low radial stability have been observed by [21]. The particle oscillation in the radial direction can lead to a dispersion of the Raman scattering intensity. [16], [17] obtained different intensity profiles for scanning in the radial and axial directions, which were attributed to the particle oscillations in the radial direction.

Aiming to conserve the acoustic levitation forces under the changing parameters temperature and pressure, this paper presents the development of a single-axial acoustic levitator for different atmospheric conditions, which is implemented in 
a Single-Droplet Optical Cell (SDOC). In a previous work the finite element method was used to determine the potential of the acoustic radiation force that acts on a levitated spherical particle [22]. This potential was used to determine the levitation positions of particles in the standing wave field. After numerical simulations and construction of the levitator and the SDOC, an image evaluation technique was used to validate the results of the simulation and the levitated spherical particle under changing atmospheres.

\section{EXPERIMENTAL APPARATUS}

\section{A. Acoustic Levitator}

Basically, an acoustic levitator consists of an ultrasonic transducer and a reflector, which are ideally separated by a distance of a multiple integer of $\lambda / 2$, where $\lambda$ is the wavelength of the acoustic wave. In this work, a single-axis acoustic levitator, consisting of a $38.5 \mathrm{kHz}$ Langevin transducer with a concave radiating surface and a concave reflector is used to levitate small spheres. A detailed description of this acoustic levitator setup is presented in [22].

The Finite Element Method was used to determine the potential of the acoustic radiation force that acts on a small sphere as a function of the distance between the transducer and the reflector, following the same procedure of [22]. Additionally, the geometry of the SDOC is added to the existing acoustic levitator model to consider wall effects like reflections of the ultrasound. All simulations were performed with a transducer operating at $38.5 \mathrm{KHz}$ with a vibration amplitude of $1 \mu \mathrm{m}$. This analysis allows the monitoring of the optimal separation distances between transducer and reflector.

The optimized separation distances for levitation at 293.15 $\mathrm{K}$ and $0.1 \mathrm{MPa}$ corresponds to $32.5 \mathrm{~mm}$. The potential for the acoustic radiation force that acts on a small sphere for this separation distance is presented in Fig. 1. The cross marks correspond to the points of minimum potential, where levitation can occur. The potential was obtained by using the theory of [23]. To show the potential that is independent of the sphere radius, the relative potential shown by [24] is illustrated in Fig. 1, which is specified by [20]:

$$
U=2 \pi r^{3}\left(\frac{\overline{p^{2}}}{3 \rho c^{2}}-\frac{\rho \overline{u^{2}}}{2}\right)
$$

where $\overline{p^{2}}$ and $\overline{u^{2}}$ are the mean square amplitudes of pressure and velocity, respectively, $r$ is the radius of the levitated particle, $\mathrm{c}$ is the fluid sound velocity and $\rho$ is the air density.

The relation between the acoustic radiation potential and the acoustic force $F$ that acts on the sphere is given by:

$$
F=-\nabla U
$$

According to equations (1) and (2) the acoustic radiation force that acts on the sphere is dependent on the particle radius. In order to obtain an acoustic radiation potential that is independent of the particle radius, a relative acoustic radiation potential is defined by [25]:

$$
\tilde{U}=\frac{U}{2 \pi r^{3}}
$$

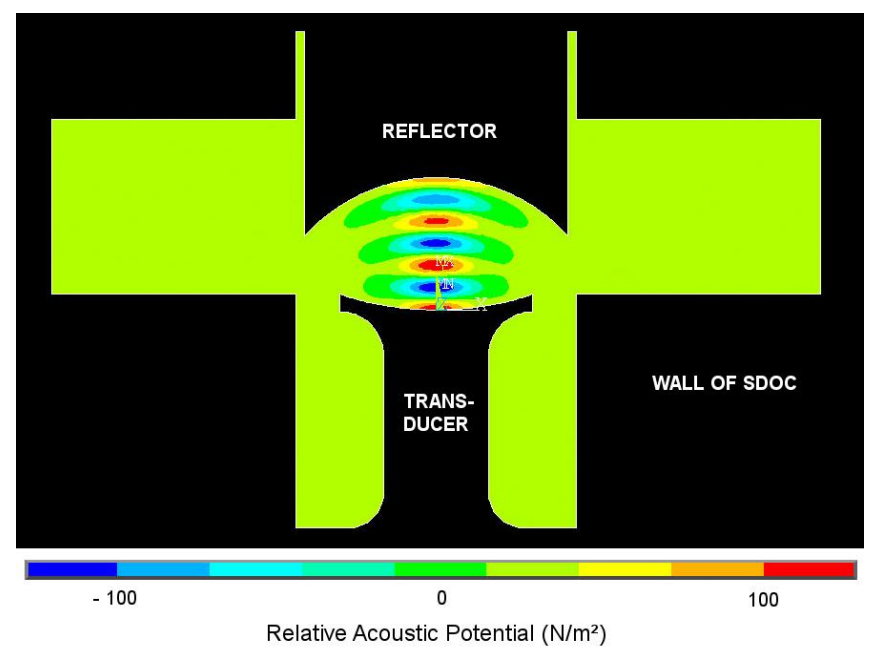

Fig. 1. Relative potential of the acoustic radiation force for a $32.5 \mathrm{~mm}$ separation distance, $1 \mu \mathrm{m}$ displacement amplitude at the transducer face an operation frequency of $38.5 \mathrm{KHz}$ and a temperature of $293 \mathrm{~K}$. The levitator is implemented in the single-droplet optical cell (SDOC).

In a similar manner a relative acoustic radiation force can be defined:

$$
\widetilde{F}=-\nabla \tilde{U}
$$

To show the potential as a function of changing atmospheres, the fluid sound velocity $\mathrm{c}_{\mathrm{m}}$ is defined as:

$$
c_{m}=\left[\sqrt{\left(\frac{T R_{m}}{M_{m}}\right) \kappa}\right] Z
$$

where $R_{m}$ is the gas constant of the used medium, $T$ is the temperature, $M_{m}$ is the molar mass of the gas, $\kappa$ is the heat capacity ratio and $Z$ is the compressibility factor. $Z$ and $\kappa$ are defined as

$$
\begin{gathered}
\kappa=\frac{c_{p}}{c_{v}} \\
Z=\frac{P}{\rho R_{m} T}
\end{gathered}
$$

with $c_{p}$ as heat capacity at constant pressure, $c_{v}$ as heat capacity at constant volume and $P$ as the pressure of the gas.

\section{B. Single Droplet Optical Cell}

The experimental apparatus used to study the acoustic levitation of particles under varying thermodynamic magnitudes in an acoustic levitator is presented in Fig. 2. In this setup, a single-axis acoustic levitator, consisting of a ultrasonic transducer with a concave radiating surface and concave reflector is implemented into a SDOC which is used to regulate the temperature and pressure of the internal atmosphere. The SDOC is made of aluminum (material 
AL7075), designed for temperatures up to $413 \mathrm{~K}$ and pressures up to $10 \mathrm{MPa}$. The material is black anodize to prevent undesirable light reflections on the inner side of the chamber. It has a nominal filling volume of $235 \pm 0.4 \times 10^{-6} \mathrm{~m}^{3}$ The autoclave has five windows mounted at the same height under different angles for Raman spectroscopy and other measuring applications. The windows are especially hardened borosilicate glasses (Maxos, Schott Auer). Liquids in form of a droplet can be induced into the acoustic levitator by punctering a membrane with a syringe. Gases can be filled into the autoclave directly from the gas bottle. During the experiment, the Langevin transducer is excited by a harmonic sine signal of $38500 \mathrm{~Hz}$ and constant voltage amplitude. This signal is generated by a $400 \mathrm{~W}$ AMMM Ultrasonic Generator (M.P. Interconsulting, Switzerland).

In order to find experimentally the distance between transducer and reflector, the acoustic levitator was calibrated by an image evaluation technique. The transducer is fixed in the experiment and an automatic positioning system (accuracy $\sim 2 \mu \mathrm{m}$ ) is used to move the reflector. The SDOC is heated by 4 heating cartridges (Hotrod HHP $500 \mathrm{~W}$, Hotset GmbH, Germany) which are controlled by a PID controller (hotcontrol C-248, Hotset GmbH, Germany). The temperature measurements are done with a PT 100 (TC Direct $\mathrm{GmbH}$, Germany) with a system accuracy of $\Delta T= \pm 0.05 \mathrm{~K}$ at $293 \mathrm{~K}$ inside the chamber. The pressure in the SDOC is established by compressed gas from bottles and measured permanently by a piezo-resistive pressure transmitter (PAA-25HTC, Keller GmbH, Germany) with an absolute accuracy of $\Delta \mathrm{P}= \pm 0.025 \mathrm{MPa}$. A camera (VRmagic $\mathrm{VR} 3+\mathrm{BW})$ is used to record the position of the levitated particle.

To maximize the contrast between the levitated sphere and the background, a LED backlight is used to illuminate the sphere. A tracking algorithm was implemented in National Instruments LabView to obtain the particle position by reaching a stable atmosphere.

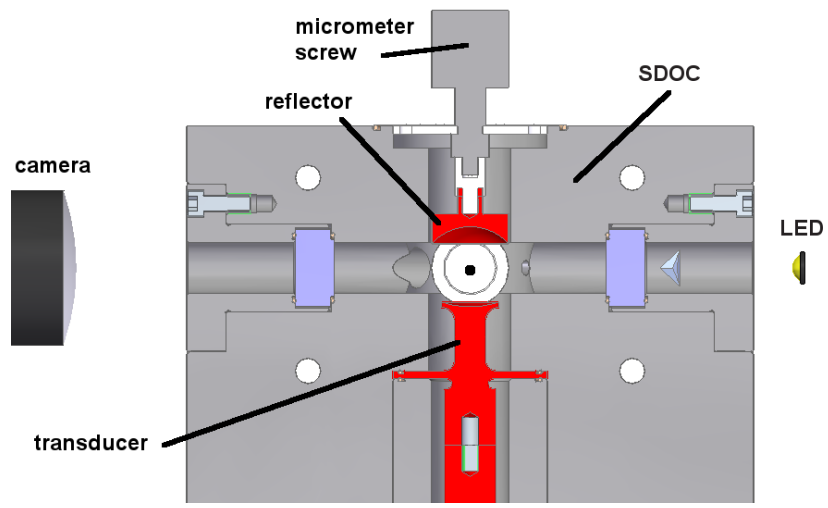

Fig. 2. The single-axis acoustic levitator with a concave radiating transducer surface and a concave reflector is implemented in a single-droplet optical cell (SDOC).

\section{RESULTS}

The distance between transducer and reflector as well as the position of the levitated sphere were analyzed under certain temperature and pressure conditions. The developed FEM method was used to simulate the optimal distance for temperatures up to $373 \mathrm{~K}$ and pressures up to $10 \mathrm{MPa}$.

\section{A. Air}

In a first step the compressibility factor and the sound velocity were calculated with the heat capacity data from [29]. The compressibility factors for air are calculated with temperatures up to $373 \mathrm{~K}$ and pressures up to $10 \mathrm{MPa}$ shown in Table I.

The fluid sound velocity for increased temperatures at constant pressures are presented in Fig. 3 . The sound velocity increases from $330 \mathrm{~m} / \mathrm{s}$ up to $410 \mathrm{~m} / \mathrm{s}$ nearly linear. The temperature in Fig. 4 is constant and the pressure gets increased up to $10 \mathrm{MPa}$. The curves of the sound velocity show a more exponential than linear behavior.

TABLE I: COMPRESSIBILITY FACTORS FOR AIR

\begin{tabular}{c|ccccccccc}
\hline \hline \multirow{2}{*}{$\begin{array}{c}\text { Pressure } \\
{[\mathrm{MPa}]}\end{array}$} & \multicolumn{10}{|c}{ Temperature [K] } \\
\cline { 2 - 10 } & 273,15 & 285,65 & 298,15 & 310,65 & 323,15 & 335,65 & 348,15 & 360,65 & 373,15 \\
\hline & \multicolumn{10}{c}{ Compressibility Factors } \\
\cline { 2 - 10 } 0.1 & 0,9994 & 0,9995 & 0,9997 & 0,9998 & 0,9999 & 0,9999 & 1,0000 & 1,0000 & 1,0001 \\
0.5 & 0,9971 & 0,9977 & 0,9984 & 0,9989 & 0,9994 & 0,9997 & 1,0001 & 1,0003 & 1,0006 \\
1 & 0,9944 & 0,9957 & 0,9970 & 0,9980 & 0,9989 & 0,9996 & 1,0002 & 1,0007 & 1,0012 \\
2 & 0,9893 & 0,9919 & 0,9945 & 0,9964 & 0,9982 & 0,9995 & 1,0008 & 1,0017 & 1,0027 \\
3 & 0,9849 & 0,9887 & 0,9926 & 0,9953 & 0,9979 & 0,9998 & 1,0017 & 1,0031 & 1,0045 \\
4 & 0,9810 & 0,9860 & 0,9911 & 0,9945 & 0,9980 & 1,0005 & 1,0030 & 1,0048 & 1,0066 \\
5 & 0,9777 & 0,9839 & 0,9900 & 0,9943 & 0,9986 & 1,0016 & 1,0046 & 1,0067 & 1,0089 \\
6 & 0,9750 & 0,9822 & 0,9894 & 0,9944 & 0,9994 & 1,0029 & 1,0065 & 1,0090 & 1,0115 \\
7 & 0,9729 & 0,9811 & 0,9893 & 0,9950 & 1,0007 & 1,0046 & 1,0086 & 1,0114 & 1,0143 \\
8 & 0,9714 & 0,9805 & 0,9897 & 0,9959 & 1,0022 & 1,0066 & 1,0111 & 1,0142 & 1,0173 \\
9 & 0,9706 & 0,9805 & 0,9905 & 0,9973 & 1,0042 & 1,0090 & 1,0138 & 1,0172 & 1,0206 \\
10 & 0,9704 & 0,9811 & 0,9918 & 0,9992 & 1,0065 & 1,0116 & 1,0168 & 1,0204 & 1,0241 \\
\hline \hline
\end{tabular}

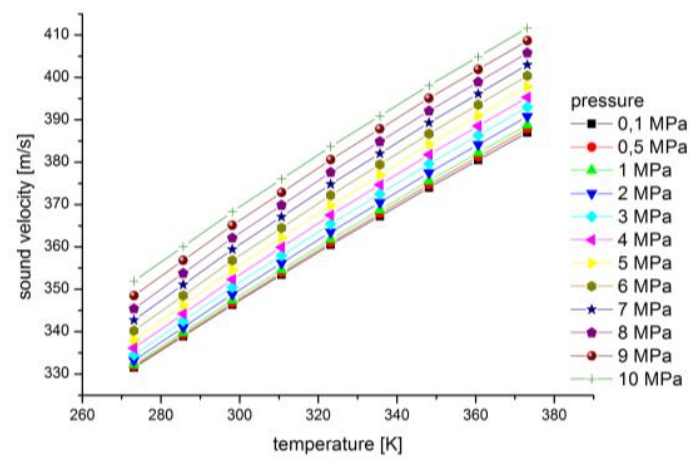

Fig. 3. Sound velocity of air under increased temperature up to $373 \mathrm{~K}$ and constant pressures.

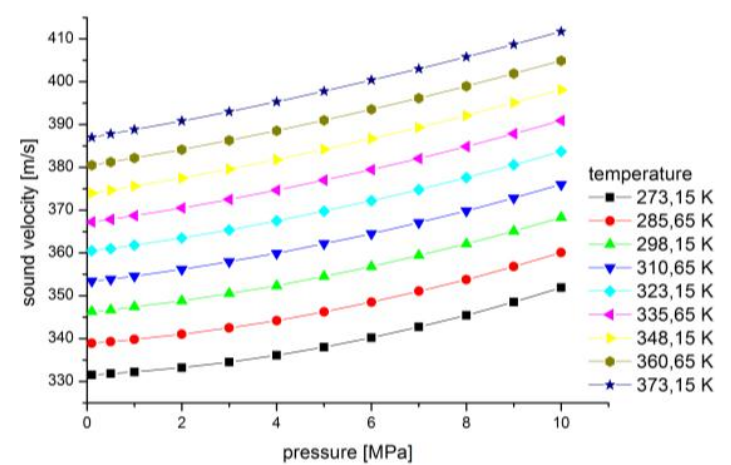

Fig. 4 Sound velocity of air under increased pressure up to $10 \mathrm{MPa}$ and constant temperatures.

To find the best separation between transducer and reflector of the developed acoustic levitator geometry under increased temperature and pressure, the FEM method was used to find the minimum potential of the acoustic radiation 
force for each thermodynamic state. Table II shows the simulated optimal distances between the transducer and reflector of the acoustic levitator for increased temperatures and pressures of air. The results show a rather more temperature influence than a pressure influence. By increasing the temperature at constant pressure of $0.1 \mathrm{MPa}$ from $273 \mathrm{~K}$ up to $373 \mathrm{~K}$ the separation has to be increased up to $5.0 \mathrm{~mm}$. By increasing the pressure up to $10 \mathrm{MPa}$ the distance has to be readjusted by $1.9 \mathrm{~mm}$ to get a stable levitation position.

TABLE II: SIMULATED SEPARATION DISTANCES FOR ACOUSTIC LEVITATION UNDER AIR ATMOSPHERE

\begin{tabular}{c|cccccccccc}
\hline \multirow{2}{*}{$\begin{array}{c}\text { Pressure } \\
{[\mathrm{MPa}]}\end{array}$} & \multicolumn{10}{|c}{ Temperatur [K] } \\
\cline { 2 - 10 } & 273,15 & 285,65 & 298,15 & 310,65 & 323,15 & 335,65 & 348,15 & 360,65 & 373,15 \\
\hline & \multicolumn{10}{c}{ Distance [mm] } \\
\cline { 2 - 11 } 0.1 & 31,60 & 32,20 & 32,80 & 33,40 & 34,00 & 34,60 & 35,20 & 35,90 & 36,60 \\
0.5 & 31,70 & 32,35 & 33,00 & 33,55 & 34,10 & 34,70 & 35,30 & 36,00 & 36,70 \\
1 & 31,80 & 32,40 & 33,00 & 33,60 & 34,20 & 34,80 & 35,40 & 36,05 & 36,70 \\
2 & 31,87 & 32,46 & 33,04 & 33,69 & 34,34 & 34,96 & 35,57 & 36,25 & 36,93 \\
3 & 32,01 & 32,58 & 33,14 & 33,84 & 34,53 & 35,15 & 35,77 & 36,44 & 37,11 \\
4 & 32,16 & 32,71 & 33,26 & 34,00 & 34,74 & 35,36 & 35,99 & 36,64 & 37,30 \\
5 & 32,30 & 32,90 & 33,50 & 34,20 & 34,90 & 35,55 & 36,20 & 36,85 & 37,50 \\
6 & 32,49 & 33,03 & 33,57 & 34,37 & 35,18 & 35,81 & 36,45 & 37,07 & 37,68 \\
7 & 32,68 & 33,22 & 33,75 & 34,58 & 35,41 & 36,06 & 36,70 & 37,29 & 37,88 \\
8 & 32,88 & 33,42 & 33,96 & 34,81 & 35,66 & 36,31 & 36,96 & 37,52 & 38,08 \\
9 & 33,09 & 33,63 & 34,18 & 35,05 & 35,93 & 36,58 & 37,24 & 37,76 & 38,29 \\
10 & 33,30 & 34,10 & 34,90 & 35,55 & 36,20 & 36,85 & 37,50 & 38,00 & 38,50 \\
\hline \hline
\end{tabular}

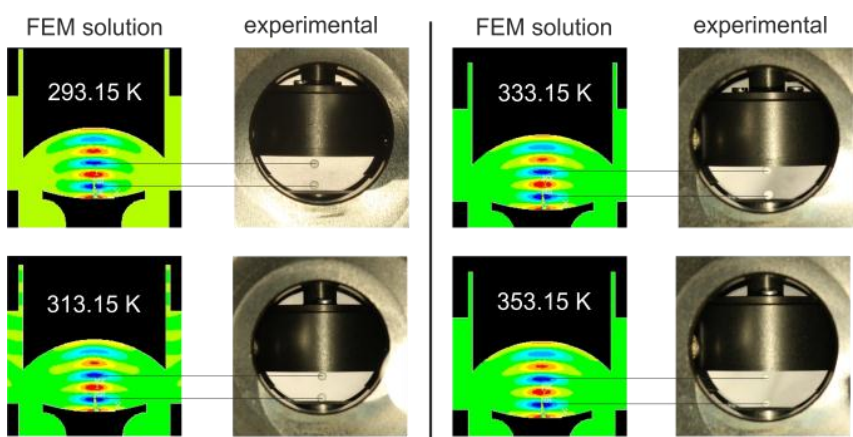

Fig. 5. Comparison between the numerical acoustic levitation potential and the experimentally obtained levitation position of small styrofoam spheres at different operation points.

To verify experimentally some simulated distances, small styrofoam spheres were placed in the levitator after determining the optimal operation distances for levitation. For a better visualisation and comparison of the experimental results and the simulated distances, the experiments are done at increased temperatures and a constant pressure of $0.1 \mathrm{MPa}$ with an opened SDOC. For high pressure and different gas verifications the chamber has to be closed and a visualization of the particle without any outer geometry relations of the acoustic levitator would be possible. During the levitation, the ultrasonic transducer was excited by a sine wave of $38500 \mathrm{~Hz}$ and the SDOC was heated up to the desired temperature. The comparison between the relative acoustic potential and the levitation position for different is presented in Fig. 5. The positions of a minimum acoustic potential are denoted by cross marks. Obviously the levitation positions of the spheres are consistent to the positions of the minimal acoustic potentials.
The simulated results are experimentally verified and are reproduced in a diagram shown in Fig. 6 where the optimized distance is plotted in correlation to temperature and pressure. With an automatic positioning system and the information of best separation distance between transducer and reflector, a stable levitation for each thermodynamic states is possible and thermodynamic investigations on particles inside the levitator are possible without any interruptions.
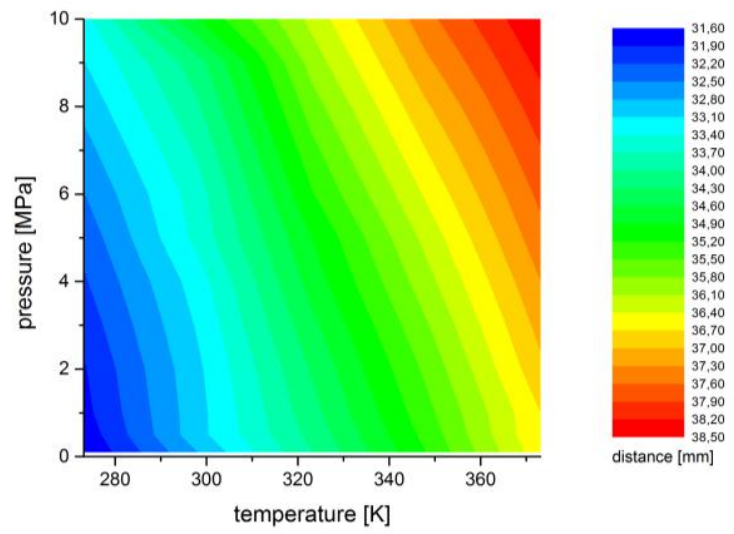

Fig. 6. Best distances between transducer and reflector of an acoustic levitator under increased temperatures and pressures of air.

TABLE III: COMPRESSIBILITY FACTORS FOR CARBON DIOXIDE

\begin{tabular}{|c|c|c|c|c|c|c|c|c|c|}
\hline \multirow{2}{*}{$\begin{array}{c}\text { Pressure } \\
{[\mathrm{MPa}]}\end{array}$} & \multicolumn{9}{|c|}{ Temperature $[\mathrm{K}]$} \\
\hline & 273,15 & 283,15 & 293,15 & 303,15 & 313,15 & 323,15 & 333,15 & 353,15 & 373,15 \\
\hline & \multicolumn{9}{|c|}{ Compressibility factor } \\
\hline 0.1 & 0,9934 & 0,9941 & 0,9948 & 0,9953 & 0,9958 & 0,9962 & 0,9966 & 0,9972 & 0,9977 \\
\hline 0.5 & 0,9660 & 0,9699 & 0,9733 & 0,9761 & 0,9786 & 0,9808 & 0,9827 & 0,9859 & 0,9884 \\
\hline 1 & 0,9300 & 0,9384 & 0,9454 & 0,9515 & 0,9566 & 0,9611 & 0,9651 & 0,9716 & 0,9767 \\
\hline 2 & 0,8498 & 0,8695 & 0,8857 & 0,8992 & 0,9105 & 0,9203 & 0,9287 & 0,9423 & 0,9529 \\
\hline 3 & 0,7518 & 0,7898 & 0,8188 & 0,8420 & 0,8611 & 0,8770 & 0,8905 & 0,9122 & 0,9288 \\
\hline 4 & & 0,6898 & 0,7409 & 0,7781 & 0,8072 & 0,8309 & 0,8505 & 0,8813 & 0,9042 \\
\hline 5 & & & 0,6419 & 0,7040 & 0,7476 & 0,7812 & 0,8082 & 0,8493 & 0,8794 \\
\hline 6 & & & & 0,6110 & 0,6795 & 0,7269 & 0,7632 & 0,8165 & 0,8542 \\
\hline 7 & & & & 0,4586 & 0,5975 & 0,6666 & 0,7151 & 0,7826 & 0,8288 \\
\hline
\end{tabular}

\section{B. Carbon Dioxide}

The effects of carbon dioxide are of significant interest in a lot of applications especially as a solvent in the field of chemical engineering.

Similar to the evaluation with air, in a first step the compressibility factor and the sound velocity of carbon dioxide were calculated with the heat capacity data from [26]. The calculated compressibility factors of carbon dioxide are listed in Table III.

The fluid sound velocity for increased temperatures at constant pressures of carbon dioxide is shown in Fig. 7. The sound velocity increases from $190 \mathrm{~m} / \mathrm{s}$ up to $300 \mathrm{~m} / \mathrm{s}$ in the gas phase of carbon dioxide. The curves of the sound velocity are shown in Fig. 8 where the temperature is constant and the pressure gets increased up to $7 \mathrm{MPa}$. The sound velocity decreases under higher pressures. These data were used to find the minimum potential of the acoustic levitation force with the described Finite Element Method in correlation of the transducer - reflector distance for a temperature range from $273 \mathrm{~K}-373 \mathrm{~K}$ and a pressure range from $0.1 \mathrm{MPa}-7 \mathrm{MPa}$ 
without calculations of the supercritical \& liquid phase.

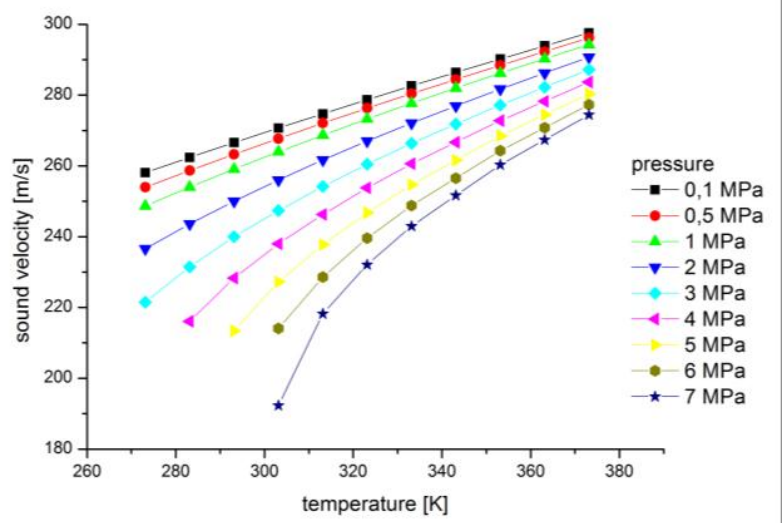

Fig. 7. Sound velocity of carbon dioxide under increased temperatures up to $373 \mathrm{~K}$ and constant pressure.

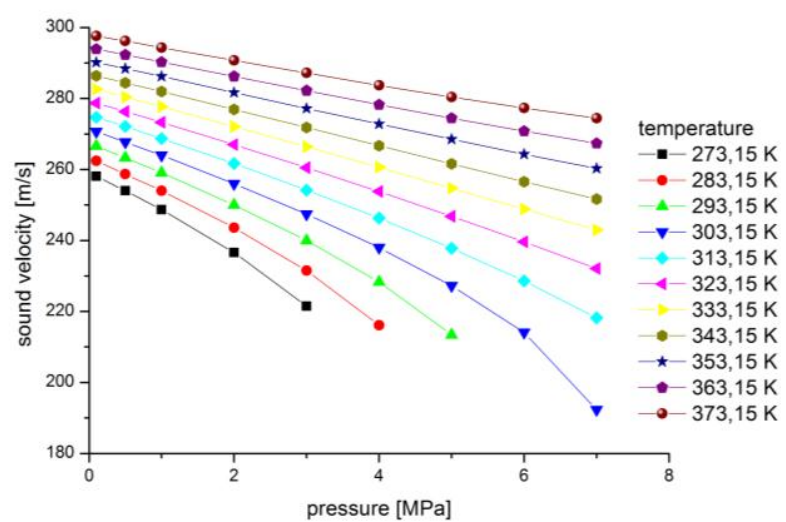

Fig. 8. Sound velocity of carbon dioxide under increased pressures up to 7 $\mathrm{MPa}$ and constant temperature.

Table IV presents the results of the simulated separation distances for an acoustic levitator with 3 levitation points under a carbon dioxide atmosphere. The ultrasonic levitator is excited by a sine wave of $38500 \mathrm{~Hz}$ and the SDOC is heated up to the desired temperature and pressure. The darkened fields represent the supercritical \& liquid phase of carbon dioxide which is not useable for acoustic levitation and not investigated in this paper. The simulated results are experimentally verified and plotted in Fig. 9 where the separation distance is in correlation to the temperature and pressure.

TABLE IV: SIMULATED SEPARATION DISTANCES FOR ACOUSTIC LEVITATION UNDER CARBON DIOXIDE ATMOSPHERE

\begin{tabular}{c|rrrrrrrrr}
\hline \hline \multirow{2}{*}{$\begin{array}{c}\text { Pressure } \\
{[\mathrm{MPa}]}\end{array}$} & \multicolumn{7}{|c}{ Temperature [K] } \\
\cline { 2 - 9 } & 273,15 & 283,15 & 293,15 & 303,15 & 313,15 & 323,15 & 333,15 & 353,15 & 373,15 \\
\hline & \multicolumn{7}{|c}{ Distance [mm] } \\
\cline { 2 - 10 } 0.1 & 25,2 & 25,5 & 25,9 & 26,3 & 26,7 & 27,1 & 27,4 & 27,9 & 28,5 \\
0.5 & 24,8 & 25,2 & 25,7 & 26,2 & 26,5 & 26,9 & 27,2 & 27,7 & 28,4 \\
1 & 24,4 & 24,8 & 25,3 & 25,7 & 26,2 & 26,6 & 26,9 & 27,6 & 28,3 \\
2 & 23,2 & 23,7 & 24,5 & 24,7 & 25,4 & 26,0 & 26,4 & 27,2 & 28,0 \\
3 & 22 & 22,8 & 23,6 & 24,3 & 24,9 & 25,4 & 25,9 & 26,8 & 27,7 \\
4 & & 21,5 & 22,4 & 22,7 & 24,0 & 24,7 & 25,4 & 26,4 & 27,4 \\
5 & & & 21,2 & 21,7 & 23,2 & 24,1 & 24,9 & 26,1 & 27,2 \\
6 & & & & 20,7 & 22,5 & 23,5 & 24,4 & 25,7 & 26,9 \\
7 & & & & 19,5 & 21,7 & 22,9 & 23,9 & 25,3 & 26,6 \\
\hline \hline
\end{tabular}

The verification of the distance between the transducer and reflector of the acoustic levitator under carbon dioxide atmosphere was done with the automatic positioning system and a camera to control the stable levitation. A visual verification of the ultrasonic levitator geometries with the simulated results is not possible because of the window geometry of the SDOC.

The trend of Fig. 9 shows a variance in the distance up to 9 $\mathrm{mm}$ to get a stable levitation position of each thermodynamic state. The non marked area shows the liquid and the supercritical phase, which are not investigated in this paper.

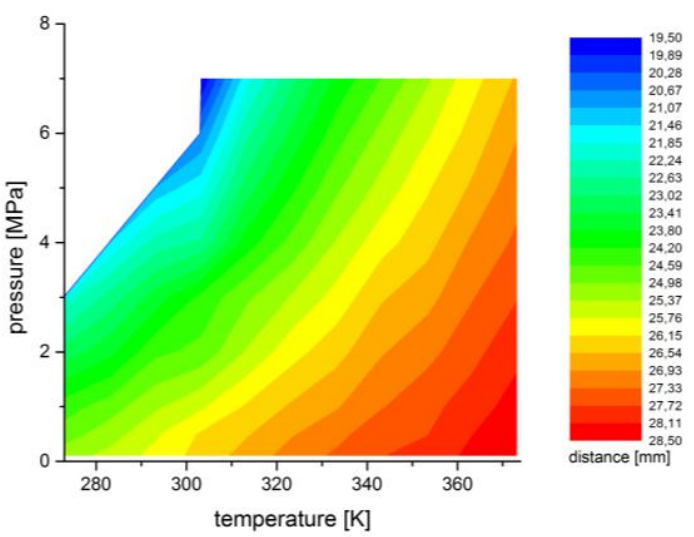

Fig. 9. Optimized distances between transducer and reflector of an acoustic levitator under increased temperatures and pressures of carbon dioxide.

\section{CONCLUSION}

The particle position and stability in a single axis acoustic levitator under varying thermodynamic conditions were analyzed. First, the Finite Element Method and the Gorkov theory were used to determine the acoustic radiation potential that acts on a small sphere in the ultrasonic levitation device. This FEM model was used to find the best distance between the transducer and reflector of an ultrasonic levitator in correlation with temperature and pressure. It was shown that the positions of minimum acoustic radiation potential agree with the levitation positions of small expanded polystyrene spheres under increased temperatures in an air atmosphere.

The main conclusion of this investigation is a good conformity of the FEM model with the experimental ultrasonic levitation. The necessity of an active distance control between transducer and reflector is inevitable to get a stable levitation position under changing thermodynamic conditions.

The FEM model can be used for the gas phase of various gases and thermodynamic changes in the surrounding atmosphere of an acoustic levitator to calculate the optimal distance between transducer and reflector. With the knowledge of the optimal levitation distances not only a stationary levitation at one special thermodynamic state can be realized. A stable levitation can be achieved for thermodynamic processes within the gas phase of the atmosphere. Changing from the gas phase of the atmosphere into the supercritical phase leads to a rigorous change in the sound velocity as well in the resulting separation of the ultrasonic levitator.

The simulated separation distances in combination with the active distance control enables the chance of novel 
investigations in the fields of thermodynamics, chemistry, biology, astrobiology, meteorology, planetary science and engineering like studying the phase equilibrium states, concentration measurements, dehydration, oxidations processes and combustion processes.

\section{REFERENCES}

[1] E. H. Trinh, "Compact acoustic levitation device for studies in fluid dynamics and material science in the laboratory and microgravity," Rev. Sci. Instrum, vol. 56, pp. 2059, 1985.

[2] S. Santesson and S. Nilsson, "Airborne chemistry: acoustic levitation in chemical analysis," Anal. Bioanal. Chem., vol. 378, pp. 1704-1709, 2004.

[3] K. C. Neuman and S. M. Block, "Optical trapping," Rev. Sci. Instrum, vol. 75, pp. 2787, 2004.

[4] M. D. Barnes, K. C. Ng, W. B. Whitten, and J. M. Ramsey, "Detection of single Rhodamine 6G molecules in levitated microdroplets," Anal. Chem., vol. 65, pp. 2360, 1993.

[5] E. G. Lierke, "Vergleichende betrachtungen zur berührungslosen positionierung von einzeltropfen in aerodynamischen, akustischen und elektrischen kraftfeldern," Forsch. Ingenieurwes, vol. 61, pp. 201, 1995.

[6] D. Gerlich, "Molecular ions and nanoparticles in RF and AC Traps," Hyperfine Interactions, pp. 293-306, 2003.

[7] M. V. Berry and A. K. Geim, "Of flying frogs and levitrons," Eur. J. Phys., vol. 18, pp. 307, 1997.

[8] K. Bücks and H. Müller, "Über einige beobachtungen an schwingenden piezoquarzen und ihrem schallfeld," Z. Phys., vol. 84, issue 1-2, pp. 75-86, 1933.

[9] L. V. King, "On the acoustic radiation pressure on spheres," Proc. Roy. Soc. Lond. Ser. A, vol. 147, 1934

[10] A. Biswas, "Thermal processing of o-terphenyl: a Raman study," Appl. Spectrosc., vol. 47, pp. 458-462, 1993.

[11] A. Biswas, "Solidification of acoustically levitated o-terphenyl crystals: A Raman study," J. Cryst. Growth, vol. 147, pp. 155, 1995.

[12] A. N. Davies, P. Jacob, A. Stockhaus, R. Kuckuk, W. Hill, R. Hergenröder, A. Zybin, and D. Klockow, "Acoustic trap for simplified micro-sample handling in laser spectroscopy," Appl. Spectrosc., vol. 54, pp.1831, 2000.

[13] L. Puskar, R. Tuckermann, T. Frosch, J. Popp, V. Ly, D. McNaughton, and B. R. Wood, "Raman acoustic levitation spectroscopy of red blood cells and Plasmodium falciparum trophozoites," Lab Chip, vol. 7, pp. $1125,2007$.

[14] B. R. Wood, P. Heraud, S. Stojkovic, D. Morrison, J. Beardall, and D. McNaughton, "A portable Raman acoustic levitation spectroscopic system for the identification and environmental monitoring of algal cells," Anal Chem, vol. 77, pp. 4955-4961, 2005.

[15] S. Santesson, J. Johansson, L. S. Taylor, I. Levander, S. Fox, M. Sepaniak, and S. Nilsson, "Airborne chemistry coupled to Raman spectroscopy,” Anal Chem, vol. 75, pp. 2177-2180, 2003.

[16] R. Tuckermann, L. Puskar, M. Zavabeti, R. Sekine, and D. McNaughton, "Chemical analysis of acoustically levitated drops by Raman spectroscopy," Anal. Bioanal. Chem., vol. 394, pp. 1433-1441, 2009.

[17] C. Esen, T. Weigel, V. Sprynchak, and G. Schweiger, "Raman spectroscopy on deformed droplets: theory and experiment," J. Quant. Spectrosc. Radiat. Transf., vol. 89, pp.79-85, 2004.

[18] A. L. Yarin, M. Pfaffenlehner, and C. Tropea, "On the acoustic levitation of droplets," J. Fluid. Mech., vol. 356, pp. 65-91, 1998.
[19] L. Yarin, G. Brenn, J. Keller, M. Pfaffenlehner, E. Ryssel, and C. Tropea, "Flowfield characteristics of an aerodynamic acoustic levitator," Phys. Fluids, vol. 9, pp. 3300, 1997.

[20] M. A. B. Andrade, F. Buiochi, and J. C. Adamowski, "Finite element analysis and optimization of a single-axis acoustic levitator," IEEE Trans. Ultrason.Ferroelectr. Freq. Control, vol. 57, pp. 469-479, 2010.

[21] S. Baer, M. A. B. Andrade, C. Esen, J. C. Adamowski, and A. Ostendorf, " Development of a single-axis ultrasonic levitator and the study of the radial particle oscillations," in Proc. AIP Conf., vol. 1433, pp. 35, 2012.

[22] S. Baer, M. A. B. Andrade, C. Esen, J. C. Adamowski, G. Schweiger, and A. Ostendorf, "Analysis of the particle stability in a new designed ultrasonic levitation device," Rev. Sci. Instr., vol. 82, pp. 105111105111-7, 2011.

[23] L. P. Gor'kov, "On the forces acting on a small particle in an acoustic field in an ideal fluid," Sov. Phys. Dokl., vol. 6, no. 9, pp. 773-775, 1962.

[24] M. Barmatz and P. Collas, "Acoustic radiation potential on a sphere inplane, cylindrical and spherical standing wave fields," J. Acoust. Soc.Am., vol. 77, pp. 928-945, 1985.

[25] W. J. Xie and B. Wie, "Dependence of acoustic levitation capabilities on geometric parameters," Phys. Rev. E, vol. 66, 026605, 2002.

[26] V. D. I. Wärmeatlas, Berechnungsunterlagen für druckverlust, wärmeund stoffübergang, ISBN 978-3540255031, Springer, 10 edition, 2006.

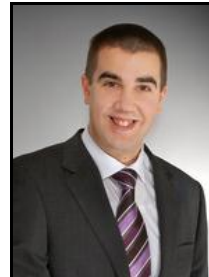

Sebastian Baer was born in Bochum, Germany, in 1982. He received the diploma degree in mechanical engineering at the Ruhr Universität Bochum in 2009. $\mathrm{He}$ is currently working toward the Ph.D. degree in mechanical engineering at the chair of applied laser technology of the Ruhr Universität Bochum. His research interests include acoustic levitation, Raman spectroscopy and optical measurement applications.

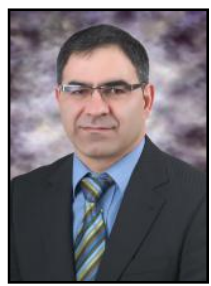

Cemal Esen studied mechanical engineering at the University of Duisburg, Germany. He received his Ph.D in mechanical engineering at the Ruhr-University in Bochum, Germany in the field of production and characterization of polymer microparticles in $1997 . \mathrm{He}$ also finished his habilitation at the Ruhr-University in Bochum in 2008. His research interests focus on development and application of spectroscopic techniques for particle analysis and optical techniques for biomedical applications.

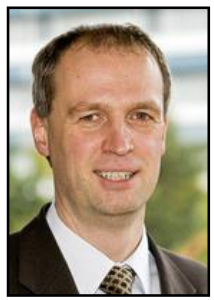

Andreas Ostendorf holds the chair of Laser Applications Technology at the Ruhr-University Bochum, Germany. He has chaired several international Laser conferences ranging from nanophotonics to laser materials processing.

His main research is on the interaction of pulsed laser radiation with biological and technical matter. He has been awarded SPIE and LIA Fellow and he is a member of the German Academic Society of Laser Technology WLT. In 2008 he was the President of the Laser Institute of America LIA. 